\title{
Collecting, Modeling, and Visualizing Network Data from Educators: A Tutorial
}

\author{
Jennifer Watling Neal and Zachary P. Neal \\ Michigan State University
}

\begin{abstract}
Understanding educators' networks can inform the field of school psychology by offering insight into how the structure of social relationships supports the implementation of schoolbased programs. However, the difficulties of collecting and modeling network data remain barriers to using network methods in school psychology. To overcome these barriers, we provide a step-by-step tutorial for collecting, modeling, and visualizing network data from educators. We draw on an example from a study designed to understand advice networks among middle and high school educators involved in implementing a system-level intervention to prevent school dropout.
\end{abstract}

\begin{abstract}
Impact and Implications Statement
Educators often work in teams to deliver services to children and adolescents within school settings. Collecting, modeling, and visualizing network data can help researchers in school psychology understand how these teams communicate or exchange advice and whether this has implications for the implementation of programs and practices. We provide a tutorial for researchers who are interested in collecting, modeling, and visualizing network data from educators.
\end{abstract}

Keywords: social network, exponential random graph model, network data collection, network visualization, educators

Network data are collected and analyzed in school psychology, developmental psychology, and education to understand how relationships are structured in schools (e.g., Carolan, 2013; Codding, 2021; J. W. Neal, 2020). Studies using school-based network data often focus on the structure of peer relationships among children or adolescents, examining the role that peer selection and influence play in psychological, social, or behavioral outcomes (J. W. Neal \& Veenstra, 2021; Sijtsema \& Lindenberg, 2018). However, network analysis can also illuminate the professional relationships of the educators that serve children and adolescents (Brown \&

Correspondence should be addressed to Jennifer Watling Neal, Michigan State University, Department of Psychology, 316 Physics Rd. Rm. 242, East Lansing, MI, 48824. Email: jneal@msu.edu. Jennifer Watling Neal (10) https://orcid.org/0000-0002-7749-8121 Zachary P. Neal (i) https://orcid.org/0000-0003-3076-4995

Funding was provided by the Spencer Foundation (\#201900052). All procedures were reviewed and determined exempt by Michigan State University's Institutional Review Board (\#STUDY00001336). All authors of this manuscript declare that they have no conflicts of interest. We would like to thank Dr. Courtenay Barrett for her collaboration on the larger project that produced the data used in this tutorial.
Poortman, 2018).

Educators serve as primary implementers of social, behavioral, and academic interventions for children and adolescents, often working in teams to deliver services within school settings. Implementing these types of interventions can often be challenging, leading educators to seek advice from one another when they encounter problems. Understanding how educators seek advice in these situations - that is, understanding educators' advice networks - can inform the field of school psychology by identifying advice-seeking strategies that might support or hinder the implementation of school-based programs (J. W. Neal, Neal, Barrett, \& Brutzman, 2020). These insights can inform school psychology practice by pointing to opportunities for improving implementation through relationship building.

We aim to facilitate the use of network methods in school psychology by providing a tutorial for collecting, modeling, and visualizing network data from educators. This tutorial is primarily intended for researchers in school psychology who are interested in applying social network methods. However, practitioners may also find the tutorial useful for interpreting the findings of existing social network studies in school psychology to inform their practice. We begin by describing the stages of network data collection including specifying the network boundary, defining the relationships in the network, and collecting the network data. Next, we provide step-by- 
step instructions for using exponential random graph modeling to understand a network's structure. Finally, we end with a discussion of visualizing network data, providing recommendations for using visualization to highlight key findings. Throughout the tutorial, we use an example from a study designed to understand advice networks among middle and high school educators in one public school district who were involved in an initiative to detect early warning signs for school dropout and implement related preventive interventions.

\section{Collecting Network Data}

\section{Why collect network data?}

Most studies in school psychology use traditional caseby-variable data, which provides information about the attributes (e.g., demographics, attitudes) of participants (e.g., teachers) in a particular sample. Quantitative analyses such as regression allow researchers to examine associations between these attributes, for example exploring how teachers' sense of belonging is related to their experiences of burnout (e.g., O’Brennan, Pas, \& Bradshaw, 2017). In contrast, network data provide information about the relationships (e.g., advice, friendship) or edges between a set of actors or nodes (e.g., teachers, students) in a system (e.g., a middle school; adams, 2020; ?). Network analysis allows researchers to characterize patterns of relationships between nodes and to understand the network's structure. For example, a network analysis might examine whether teachers' sense of belonging is related to their relationships with other teachers (Bjorklund Jr \& Daly, 2021). Therefore, researchers may want to collect network data if they have research questions that focus on relational predictors, outcomes, or processes.

Although collecting network data is similar to collecting traditional case-by-variable data, there are important differences given the relational nature of network data (e.g., adams, 2020). We illustrate these differences using an example that involves collecting network data from educators in one school district who were involved in an early warning signs (EWS) initiative. EWS is implemented by teams of school administrators and staff and is aimed at preventing school dropout among at-risk middle and high school students (Faria et al., 2017). Although various forms of EWS are implemented in U.S. public schools (U.S. Department of Education, 2016), educators in our study were implementing a version that included seven steps for using data to identify, intervene with, and monitor the outcomes of students at risk of dropping out of school (Therriault, O'Cummings, Heppen, Yerhot, \& Scala, 2017). All study procedures were reviewed and determined exempt by our university's institutional review board. We obtained active informed consent from all participants, asking them to indicate their agreement to participate by clicking a button on a web-based form.

\section{Specifying the Boundary}

Table 1 provides an overview of the stages and key considerations involved in network data collection. Case-byvariable data collection relies on drawing a sample of cases from a larger population. However, because network data should provide information about the relationships between all actors in a system (e.g., classroom, district), it is necessary to collect data on all members of the system. Therefore, the first stage of network data collection involves specifying the boundary of the system by determining which individuals should be included as nodes (adams, 2020). Boundary specification is important because omitting key nodes from data collection distorts the structure of the network and biases network analyses (Laumann, Marsden, \& Prensky, 1989). The process of specifying the boundary is straightforward in situations where complete membership rosters exist for all individuals in a particular system (e.g., all teachers in a middle school). When the system's boundaries are more difficult to delineate, it is important for researchers to clearly outline the procedures determining who counts as part of the system and who does not.

In our example, we were interested in understanding the relationships of all educators who were involved in implementing EWS within a school district. Following the steps for implementing EWS (Therriault et al., 2017), the middle school and high school established teams that included teachers, specialists such as school counselors, and administrators. These EWS teams served as the system for our network, and we included all individuals who were part of these teams as nodes in our network, which resulted in a network boundary that included 17 educators across both the middle and high school. School principals provided EWS team rosters.

\section{Defining the Relationships}

Once the network boundary is specified, the next stage in collecting network data involves defining the relationships in the network. Network studies can focus on many different types of relationships including communication (e.g., advice Borgatti \& Cross, 2003), friendship (Kitts \& Leal, 2021), affective relationships (e.g., like, trust, dislike, conflict; Harrigan, Labianca, \& Agneessens, 2020), and affiliation (e.g., hanging out, spend time with). It is important to consider the study's research question and to select a type of relationship that is conceptually relevant. For example, if a researcher is interested in how networks influence teachers' sense of school belonging, informal friendships or an affective relationship like trust may be most relevant. However, if a researcher is interested in understanding the role of networks in facilitating intervention implementation, more formal communication relationships like advice exchange may be more relevant.

In addition to defining the type of relationship, it is also 
Table 1

Stages of Network Data Collection

\begin{tabular}{llll}
\hline Stages & What does it involve? & Why is it important? & Key considerations \\
\hline $\begin{array}{lll}\text { Specifying } \\
\text { the Boundary }\end{array}$ & $\begin{array}{l}\text { Specifying the boundary } \\
\text { of the network system by } \\
\text { determining who should }\end{array}$ & $\begin{array}{l}\text { distort the resulting net- } \\
\text { be included as nodes. }\end{array}$ & $\begin{array}{l}\text { Does a membership roster exist that can be } \\
\text { used to help specify the boundary? }\end{array}$ \\
& & $\begin{array}{l}\text { If not, what procedures can be used to de- } \\
\text { termine who should count as part of the net- } \\
\text { work? What is the rationale for including or } \\
\text { excluding certain nodes? }\end{array}$
\end{tabular}

Defining the Defining the type and na- The relationships in the Relationships ture of the relationship(s) network data should be in the network. conceptually relevant to the study's research question.

Collecting the Recruiting participants Recruitment is critical Network Data and collecting network because network andata via surveys, in- alytic techniques are terviews, observations, sensitive to missing data. or archival data. For For surveys or intersurveys or interviews, it views, name generator is customary to collect questions operationalize network data using name the relationships in the generator questions. network.
- What type(s) of relationships should be collected?

- Should the relationships be directed or nondirected?

- Should the relationships be binary or weighted?

- What recruitment strategies can be used to encourage high response rates? Are resulting response rates high enough to ensure accurate network analysis?

- For surveys or interviews, what name generator(s) should be used to collect network data? Should a roster or free recall method be used? Is it possible to allow unlimited choices? necessary to define the nature of the relationship. Relationships can be directed or non-directed. Directed relationships are commonly employed for relationship types that imply the flow of something through the network (e.g., advice). For example, person $i$ might seek advice from person $j$, but not vice versa. In contrast, non-directed networks are often employed for relationship types where directionality is not relevant or possible (e.g., spending time with). For example, it is impossible for person $i$ to spend time with person $j$, but not vice versa. Relationships can also be defined as binary or weighted. Binary relationships indicate whether a relationship is present or absent, whereas weighted relationships provide additional information about the strength of the tie. For example, network studies of friendship could focus on whether or not person $i$ is friends with person $j$ (i.e., binary), or could focus on how close person $i$ feels toward person $j$ (i.e., weighted).

In our example, we were interested in understanding information exchange on the EWS team; therefore, we choose to focus on directed advice relationships. Additionally, because we were interested in understanding where educators sought implementation advice, but not how often they sought such advice, we defined these relationships as binary rather than weighted.

\section{Collecting the Network Data}

After specifying the network boundary and defining the relationships, data collection can begin. Because network analyses are sensitive to missingness (adams, 2020; J. W. Neal, 2008), many network analytic techniques require higher response rates above $80 \%$ or specialized methods for imputing missing data (e.g., Huisman, 2009; Krause, 2019). This makes recruitment efforts particularly important when collecting network data. Common recruitment strategies for boosting response rates include monetary incentives and weekly email reminders or follow-up phone calls to nonrespondents (Z. Neal, Neal, \& Piteo, 2020), with recent work among educators suggesting that follow-up phone calls can double response rates (Z. P. Neal \& Neal, 2022).

Network data can be collected using surveys, interviews, observations, and archival data (adams, 2020), with surveys and interviews being particularly common in school settings 
(J. W. Neal, 2020). When using these methods, it is customary to provide a name generator question that asks respondents to identify others with whom they have the relationship of interest. These questions provide a method of operationalizing the relationships that were defined in the previous stage. For example, a researcher interested in collecting network data on educators' trust relationships in a district might ask: Among others in your district, who do you trust? If relationships are weighted, follow-up questions can be used to assess strength or frequency (e.g., On a scale of 1 (not at all) to 5 (very much), to what extent do you trust X?). When asking name generator questions, respondents may be asked to select from a roster of nodes within the network boundary or may be asked to provide names via free recall (adams, 2020). When providing a roster is feasible, this method can help avoid errors of omission. However, whether the roster or free recall method is used, it is essential that respondents be allowed to select or provide an unlimited number of responses Holland and Leinhardt (1973).

In our example, we aimed to collect network data from all 17 EWS team members using a web-based survey between February 12, 2019 and March 26, 2019. We initially invited educators to participate in our study using an invitation email that included a personalized greeting as well as information about the survey contents, estimated time for completion (i.e., 20 minutes), and survey incentive. All participating educators received a \$10 Amazon.com gift card for their participation. We followed our initial invitation with weekly personalized reminder emails to non-respondents. Of the 17 educators initially recruited for participation, one did not respond and one indicated that they did not participate in the implementation of EWS steps. These individuals were excluded from the resulting network data, yielding a final response rate of $88.2 \%$. Among the 15 participating educators, all were White and all but one were women; for this reason, race and gender were not part of our analysis.

We used the following name generator question to collect binary, directed network data about respondents' advice seeking about the EWS steps: "In the last year, when you faced a challenge implementing the steps of EWS, who did you go to for advice?" We used a free recall method where respondents named their choices spontaneously, and we allowed respondents to provide as many names as they wished. We matched the first and last names provided by respondents to the names of educators in our network boundary. Our respondents sought advice from between 0 and 7 other team members $(M=4)$.

\section{Modeling Network Data}

Modeling network data can provide insight into the forces that led to the formation of an observed network. That is, it can help us understand why some relationships formed, but others did not. Specifically, in our example, it can help us answer the following research question: How did EWS team members choose whom to ask for advice when they encountered implementation challenges? This is an important question because effective implementation of a complex new intervention requires ensuring that school staff, including school psychologists, are able to get implementation advice when they need it. Different modeling frameworks exist for different types of network data. Because our network is cross-sectional, an exponential random graph model (ERGM; Robins, Pattison, Kalish, \& Lusher, 2007) is the most appropriate approach, but stochastic actor-oriented models (SAOM; Snijders, 2017) are more appropriate for modeling longitudinal network data.

Although exponential random graph modeling may sound complicated, it is nearly identical to logistic regression in most respects. An ERGM is designed to predict whether or not a relationship is present in the network (the binary dependent variable) based on one or more independent variables. In our example, we use an ERGM to predict whether person $i$ sought advice about EWS from person $j$ (our dependent variable). We make this prediction based on several independent variables including characteristics of individuals (i.e., node effects) such as their role at the school, characteristics of the relationship between two individuals (i.e., dyadic effects) such whether they work in the same school building, and characteristics of the entire system (i.e., structural effects) such as whether two people tend to seek advice from the same third person. Like a logistic regression, the coefficient estimates produced by an ERGM can be interpreted as log odds. The primary difference between ordinary logistic regression and ERGM is the way they estimate the coefficients' standard errors: the former uses parametric statistical methods that assume each observation is independent, while the latter uses Monte Carlo methods that do not require assuming the network members are independent (which, clearly, they are not). This alternative approach to estimation also means that ERGMs can be used to analyze even very small networks containing only four or five members (Vega Yon, Slaughter, and de la Haye, 2020).

\section{Preparing the data}

An ERGM can be estimated in R ( $\mathrm{R}$ Core Team, 2020) using the statnet package (Handcock, Hunter, Butts, Goodreau, \& Morris, 2008). In this section we provide a step-by-step illustration of an ERGM analysis using R. Appendix A shows each $R$ command in boldface and the resulting $\mathrm{R}$ output in plainface. Throughout this illustration we refer to specific lines in the appendix using square brackets. All the code and data necessary to repeat this example is also available at https://osf.io/unyxh/.

Estimating an ERGM requires two types of data, relational data and node data. Our relational data takes the form of a square matrix with a row and column for each partici- 
Table 2

\section{Relational Data}

\begin{tabular}{|l|l|l|l|l|l|l|l|l|l|l|l|l|l|l|l|}
\hline & $\mathbf{A}$ & $\mathbf{B}$ & $\mathbf{C}$ & $\mathbf{D}$ & $\mathbf{E}$ & $\mathbf{F}$ & $\mathbf{G}$ & $\mathbf{H}$ & $\mathbf{I}$ & $\mathbf{J}$ & $\mathbf{K}$ & $\mathbf{L}$ & $\mathbf{M}$ & $\mathbf{N}$ & $\mathbf{O}$ \\
\hline $\mathbf{A}$ & 0 & 1 & 0 & 0 & 0 & 1 & 0 & 1 & 1 & 0 & 1 & 1 & 0 & 1 & 0 \\
\hline $\mathbf{B}$ & 0 & 0 & 0 & 0 & 0 & 0 & 0 & 0 & 0 & 0 & 0 & 0 & 0 & 0 & 0 \\
\hline $\mathbf{C}$ & 0 & 0 & 0 & 0 & 0 & 0 & 0 & 1 & 1 & 0 & 1 & 0 & 0 & 0 & 0 \\
\hline $\mathbf{D}$ & 0 & 0 & 0 & 0 & 0 & 1 & 0 & 0 & 0 & 0 & 0 & 0 & 0 & 0 & 0 \\
\hline $\mathbf{E}$ & 0 & 1 & 0 & 0 & 0 & 1 & 0 & 0 & 0 & 0 & 0 & 1 & 0 & 1 & 0 \\
\hline $\mathbf{F}$ & 1 & 1 & 0 & 0 & 0 & 0 & 0 & 0 & 0 & 1 & 0 & 1 & 0 & 1 & 0 \\
\hline $\mathbf{G}$ & 0 & 0 & 0 & 0 & 0 & 0 & 0 & 1 & 1 & 0 & 1 & 0 & 0 & 0 & 1 \\
\hline $\mathbf{H}$ & 0 & 0 & 0 & 0 & 0 & 1 & 0 & 0 & 1 & 0 & 0 & 0 & 0 & 1 & 1 \\
\hline $\mathbf{I}$ & 0 & 0 & 0 & 0 & 0 & 0 & 0 & 1 & 0 & 0 & 1 & 0 & 0 & 0 & 1 \\
\hline $\mathbf{J}$ & 1 & 0 & 0 & 1 & 0 & 1 & 0 & 1 & 0 & 0 & 0 & 1 & 0 & 1 & 0 \\
\hline $\mathbf{K}$ & 0 & 0 & 0 & 0 & 0 & 0 & 0 & 1 & 1 & 0 & 0 & 0 & 0 & 0 & 1 \\
\hline $\mathbf{L}$ & 1 & 0 & 0 & 0 & 0 & 1 & 0 & 1 & 0 & 0 & 0 & 0 & 0 & 1 & 0 \\
\hline $\mathbf{M}$ & 0 & 1 & 0 & 0 & 0 & 0 & 0 & 1 & 1 & 0 & 1 & 0 & 0 & 0 & 1 \\
\hline $\mathbf{N}$ & 1 & 1 & 0 & 1 & 1 & 1 & 0 & 0 & 0 & 1 & 0 & 1 & 0 & 0 & 0 \\
\hline $\mathbf{O}$ & 0 & 1 & 0 & 0 & 0 & 0 & 0 & 1 & 1 & 0 & 0 & 0 & 0 & 0 & 0 \\
\hline
\end{tabular}

pant (see Table 2). In this matrix, a 1 indicates that the row participant sought advice from the column participant, while a 0 indicates they did not. It contains only $0 \mathrm{~s}$ and $1 \mathrm{~s}$ because our network is unweighted, and the entry in row $i$ and column $j$ does not necessarily match the entry in row $j$ and column $i$ because our network is directed. Our node data take the form of a case-by-variable dataset; each row represents a participant and each column represents a variable (see Table 3). These data include four variables: the participant's building (high or middle), the participant's role (teacher, specialist, or admin), the number of EWS's seven steps the participant engages in, and the number of five common challenges the participant reported encountering when implementing EWS. To preserve the participants anonymity, both datasets use the capital letters A-O to identify individuals. The relational data can be loaded into $R$ as a matrix object called matrix using line [1], and the node data can be loaded as a dataframe object called vars using line [2].

Using these data to estimate an ERGM requires first installing and loading the statnet package using lines [3-4]. The statnet package also requires that these data be combined into a single network object. Line [5] transforms the matrix into an object called network that statnet recognizes as a directed network, while lines [6-7] add each variable to this network object, treating them as attributes of the network's members.

\section{Estimating a null model}

The specification of an ERGM is similar to any regression-like model and includes a dependent variable that is predicted as a function of an intercept and set of covariates.
Table 3

\begin{tabular}{lllll} 
Node Data & & & \\
\hline participant & building & role & steps & challenges \\
\hline A & high & specialist & 7 & 1 \\
B & high & admin & 5 & 2 \\
C & middle & teacher & 4 & 3 \\
D & high & specialist & 5 & 2 \\
E & high & specialist & 7 & 3 \\
F & high & specialist & 7 & 2 \\
G & middle & teacher & 5 & 4 \\
H & middle & admin & 7 & 5 \\
I & middle & specialist & 7 & 3 \\
J & high & specialist & 7 & 2 \\
K & middle & specialist & 4 & 2 \\
L & high & specialist & 6 & 2 \\
M & middle & teacher & 7 & 5 \\
N & high & admin & 7 & 1 \\
O & middle & admin & 4 & 3 \\
\hline
\end{tabular}

Normally, these covariates would be selected a priori to test hypotheses derived from theory and past research. However, in this example, we build the model in stages to illustrate the types of covariates that can be included, the hypotheses they test, and their impact on model fit. The dependent variable is the network, or more specifically or presence and absence of each relationship in our network, and the intercept term in an ERGM is specified using edges. Thus, we begin by estimating a null or intercept only model using line [10] and summarizing its estimates using line [11], which yields the output on lines [12-15].

The coefficient associated with the edges term $(-0.9397)$ is difficult to interpret directly, but we can find its corresponding probability in the logistic distribution using line [16]; the result on line [17], 0.28 , is equal to the density of our network. Substantively, this indicates that in the absence of any other information (because this model contains no covariates), we would predict there is a $28 \%$ chance one EWS team member will seek advice from another EWS team member. However, this prediction is based on very little information, so this null model probably does not fit our data well. The AIC and BIC reported on line [15] provide overall indices of model fit, where smaller values indicate better fitting models. As we include additional model terms that correspond to factors associated with advice seeking, we expect the model fit to improve from these starting values.

Model fit can also be evaluated by examining a goodnessof-fit plot using line [18]. Figure 1 shows several goodnessof-fit plots for our null model, with each panel referring to a particular characteristic of the network. The thick black line in each panel shows the value of the corresponding characteristic in our observed network. For example, the left panel 


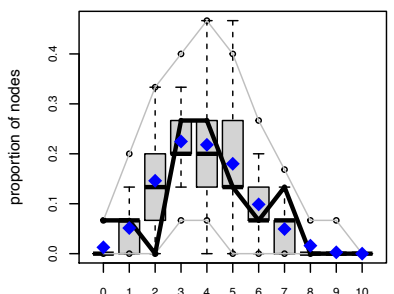

odegree

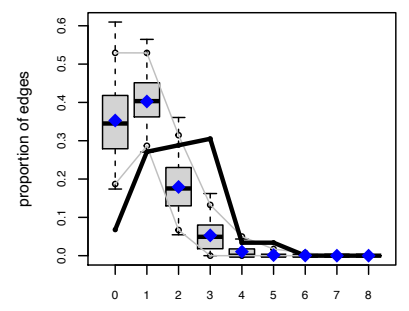

edge-wise shared partners

Figure 1

Null model goodness-of-fit.

indicates that in our network about $20 \%$ of the people have an in-degree of 0 (i.e., no one seeks advice from them), and no one has an in-degree of 3 (i.e., 3 people seek advice from them). The two thin gray lines in each panel show the $25^{\text {th }}$ and $75^{\text {th }}$ percentile of this characteristic in networks simulated from our model estimates. In a well-fitting model, the thick black line should be between the thin gray lines, indicating that our model estimates describe a process of network formation that would lead to networks similar to our observed network. However, there are several instances in Figure 1 where the thick black line lies outside the thin gray lines (e.g., in-degree $=3$ ), indicating that our null model does not fit the data well. This is expected because our null model does not contain any covariates that may help explain relationship formation; however, adding covariates to the model can make it more informative and improve its fit.

\section{Adding individual characteristics}

We might hypothesize that characteristics of the EWS team members are associated with their patterns of advice seeking. We can include individual characteristics as covariates using the nodefactor term for categorical covariates and nodecov term for continuous covariates, estimating a more complex model using lines [19-23]. The two nodefactor terms test the hypotheses that where an individual works (i.e., in the middle school or high school) and what they do (i.e., teacher, specialist, or administrator) is associated with their likelihood of exchanging advice with other EWS team members. Similarly, the nodecov terms test the hypotheses that the number of EWS steps a team member is involved in implementing, and the number of implementation challenges they have encountered, is associated with their likelihood of exchanging advice.

After estimating this model, we summarize it using line [24], which yields the output on lines [25-33]. The significant and positive nodefactor.building.middle indicates that EWS team members at the middle school are more likely to exchange advice than team members at the high school, which are the omitted category. Similarly, the significant and positive nodefactor.role.admin coefficient indicates that administrators are more likely to exchange advice than teachers, which are the omitted category. These findings are consistent with our expectations that middle school staff would be more engaged because the focus is on identifying early warning signs, and that administrators would be more engaged because EWS is a systemwide implementation. The significant and opposite-signed nodecov. steps and nodecov. challenges coefficients indicate that EWS team members are more likely to exchange advice about EWS when they are engaged in more EWS steps, and when they have experienced fewer challenges implementing EWS. Again, this is consistent with our expectations that team members who are more involved with EWS would need more advice, and team members who encounter challenges would have less advice to give. To interpret the magnitude of these coefficients, we can insert specific covariate values into our estimated regression equation, then find the corresponding probability in the logistic distribution again using the plogis function in line [34]. For example, these model estimates suggest that two EWS team members who are both at the middle school $(1.0098 \times 2)$, who are both administrators $(1.2641 \times 2)$, who both are engaged in implementing all 7 EWS steps $(0.4471 \times 2 \times 7)$, and who have not experienced any challenges implementing EWS $(-0.3406 \times 2 \times 0)$, have a nearly $98 \%$ probability of exchanging advice about EWS with each other. By incorporating these individual characteristics, the model is more informative and fits the data better than the null model (AIC declined from 251.4 to 234.2).

\section{Adding dyad characteristics}

Although EWS team members' individual characteristics may shape how much they participate in advice sharing, we might also hypothesize that advice sharing is more likely between pairs of team members who are similar, which we can test by including nodematch terms in a new model estimated by lines [36-42]. The two nodematch terms test the hypotheses that whether two EWS team members work in the same building, or occupy the same role, is associated with the likelihood they exchange advice. In the network literature, this phenomenon is known as homophily, and is captured by the notion that "birds of a feather flock together" (McPherson, 
Smith-Lovin, \& Cook, 2001).

Again, after estimating this model, we summarize and interpret its estimated coefficients and model fit using line [43], which yields the output on lines [44-54]. Controlling for the individual characteristics included in model 2 , which remain statistically significant, we observe evidence of homophily with respect to building. Specifically, the significant and positive nodematch.building coefficient indicates that EWS team members are more likely to exchange advice with other team members in their own building than with team members in a different building. In contrast, the non-significant nodematch. role coefficient indicates that sharing roles is not associated with exchanging advice. These findings are consistent with our expectations that advice about implementing a program will often be building-specific and shared among staff in that building, but as a multi-faceted intervention, advice about implementing EWS is exchanged by staff playing different roles within that building. Adding these dyadic characteristics has further improved the model fit (AIC declined from 234.2 to 176.4).

\section{Adding structural characteristics}

The characteristics of people in a network frequently influence the formation of relationships; however, the presence of some relationships can influence the formation of other relationships. A key strength of an ERGM is its ability to test hypotheses about how relationships can influence each other, and in our example, to examine how some advice exchanges might influence the likelihood of other advice exchanges. In a final model, we add a series of structural terms to explore this possibility, estimating the model using lines [55-64]. Figure 2 graphically summarizes the hypotheses that each of these structural terms are designed to test. In these diagrams, circles represent people, the dashed line represents the hypothesized advice-seeking relationship, and the solid lines represent advice-seeking relationships that we might observe. First, the mutual term tests the hypothesis that $i$ is more likely to seek advice from $j$ if $j$ seeks advice from $i$, and thus that two network members will engage in a mutual exchange or reciprocity. Second, the gwesp term hypothesizes that $i$ is more likely to seek advice from $j$ if $i$ seeks advice from a third person $(k)$ that also seeks advice from $j$, and thus hypothesizes that individuals will seek to "go straight to the source" or increase transitivity. Finally, the idegree 1.5 term hypothesizes that $i$ is more likely to seek advice from $j$ if many others (e.g., $k, l$ ) seek advice from $j$ also, and thus hypothesizes that individuals will seek advice from the most widely used sources or that relationship formation will be guided by popularity. Although there are many other structural effects that could be included to test other hypotheses, these are among the most common.

After estimating this complete model, we summarize and interpret its coefficients, and inspect the model's fit using

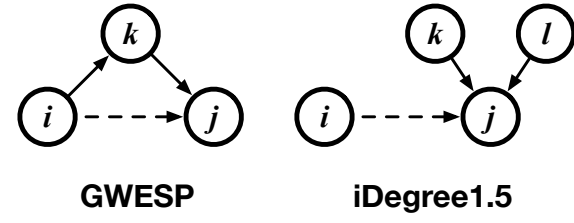

Figure 2

Hypotheses tested by selected ERGM structural effects.

line [65], which yields the output on lines [66-79]. Controlling for the individual and dyadic characteristics included in model 3, which remain statistically significant, we observe evidence that advice-seeking relationships influence each other. The significant and negative mutual coefficient indicates that EWS team members' do not reciprocate advice seeking. Although reciprocity may be common for social relationships such as friendship, this finding is consistent with expectations that advice-seeking relationships are not reciprocal because some people need information and other people have information. The significant and positive gwesp. fixed. $\boldsymbol{0} .7$ coefficient indicates that EWS team members aim to seek advice directly from their source's source. This finding is consistent with our expectation that EWS team members will try to get advice faster and with less distortion by avoiding intermediaries. Finally, the significant and positive idegree 1.5 coefficient indicates that EWS team members are more likely to seek advice from popular team members to whom others also turn. This is consistent with our expectation that EWS teams will include one or more members perceived to be particularly good sources of advice about implementation. Adding these structural terms has further improved the model's fit (AIC declined from 176.4 to 160.6 ), but re-examining the goodness-of-fit plots using line [80] provides more detailed information about fit. In these plots (see Figure 3), the thick black line (our network's characteristics) is always between the thin gray lines (simulated networks' characteristics), indicating that our final model fits the observed data very well.

\section{Drawing conclusions}

Having confirmed our final model's goodness-of-fit, we draw conclusions from its estimates and identify their implications for practice. First, with respect to school buildings, we found that implementation advice exchanges are more common among middle school staff, which is consistent for an intervention focused on identifying early warning signs. However, we also found that these exchanges are concentrated within buildings, which may limit opportunities to coordinate the program's implementation across the two buildings, which is especially important as students exhibiting these warning signs transition from middle school to high school. Second, with respect to EWS team members' roles, 

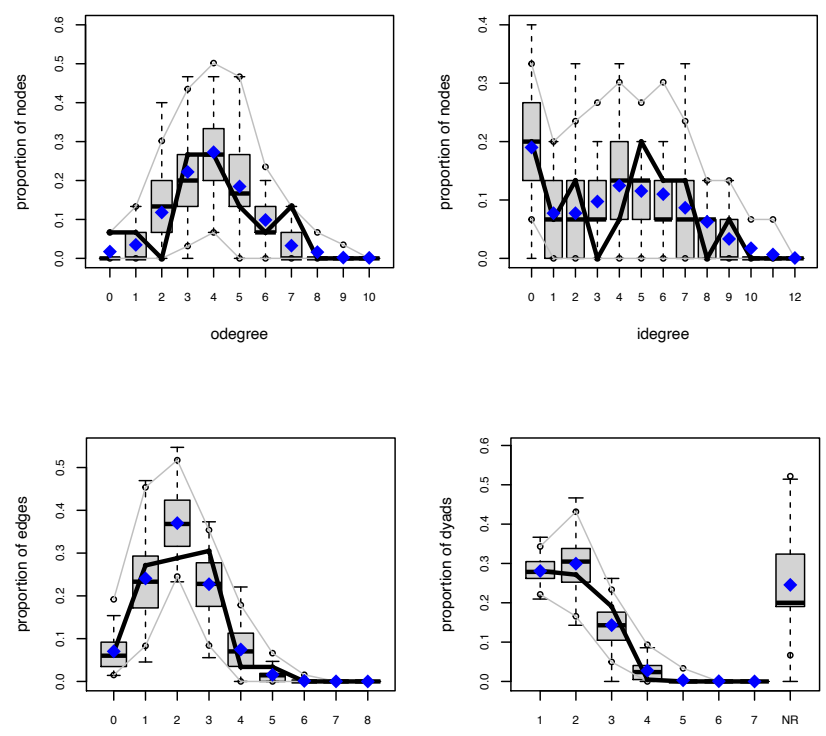

edge-wise shared partners

Figure 3

Final model goodness-of-fit.

we found that administrators are more likely to seek or give advice than teachers which may simply reflect the nature of their job. However, it may also highlight a risk of implementation burnout among administrators, and a risk that teachers are isolated from useful advice about implementation. Third, with respect to team members' engagement in EWS implementation, we found weak (i.e., trend-significant, $p=0.078$ ) evidence that experiencing implementation challenges was associated with fewer advice exchanges. Because these data are cross-sectional, we are unable to determine whether experiencing challenges leads team members to withdraw, or whether isolation from advice exchanges creates challenges for them, but this finding highlights a possible barrier to implementation. Finally, with respect to network structure, we find that team members tend to seek advice directly from popular advice-givers. On the one hand, this may be beneficial because it reduces the number sources an advice-seeker needs to consult, but on the other hand, it may place the burden of advice-giving on just a few individuals.

\section{Visualizing Network Data}

Network studies often include a visualization of the network. Although network visualizations can be a useful way to communicate key findings, they can sometimes be uninformative or even misleading. The decision about whether to include a network visualization should be based on its usefulness as an illustration of findings from the analysis. Likewise, decisions about how a visualization looks should focus on highlighting key findings clearly. In this section, we show how some strategic decisions about what to include can yield a network visualization that illustrates the findings from our example analysis.

\section{Preparing the data}

Although the statnet package offers some network visualization capabilities, the options are more robust in the igraph package (Csardi, Nepusz, et al., 2006). The igraph package can be installed and loaded using lines [81-82]. Our relational data are already stored in an object called matrix, but must be converted into a format that igraph recognizes as a network object that we call network using line [83]. Finally, before constructing the visualization itself, we create a couple auxiliary variables that will be useful. Lines [84-85] create lists of the IDs of EWS team members working in the high school and middle school, respectively, and store these lists in objects called high and middle. Lines [86-87] create a new variable in our vars dataset called color, and sets the value of this variable to gray90 (the $\mathrm{R}$ name for light gray) for all team members, but changes it to red for team members who are administrators.

\section{Designing the visualization}

Network visualizations are created by igraph using the plot command, which by default simply draws each network member as a labeled circle and each relationship as a black line, but offers many options to customize the design. Plotting the network using lines [88-96] adds several custom options to more clearly highlight key features of the network, and yields the visualization shown in Figure 4. We first describe what these options do, then explore how these design options help communicate some of our key findings. The mark. groups option draws a shaded polygon around the nodes representing staff working in each building. The edge.color option draws each relationship with a black line that is $33 \%$ transparent so that overlapping lines are easier to see, while the edge.arrow.size option draws small arrowheads on the lines to indicate who asked whom for advice. The vertex.color option draws each node using the color in the color variable, and thus draws most EWS team members as light gray circles, but draws administrators as red circles. The vertex. size option resizes each node based on its total number of advice exchange relationships (i.e., its degree), so that nodes representing team members who exchange advice with many others will appear larger. The layout option arranges the nodes so that team members who exchange advice will be close together, while team members who do not directly exchange advice will be further apart. The final two options, mark. border and vertex. frame. color ensure that no borders are drawn around the building polygons or nodes, which eliminates visual clutter and leads to a cleaner visualization. 


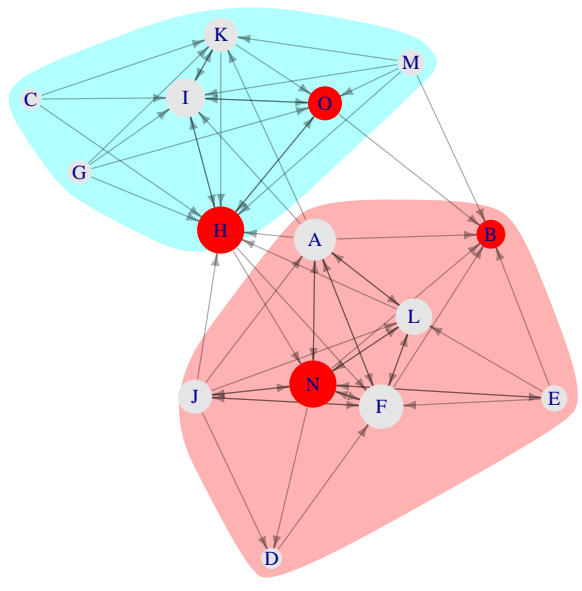

\section{Figure 4}

\section{Visualization of the EWS advice-seeking network.}

Although our ERGM revealed many potentially interesting and statistically significant influences on where EWS team members seek implementation advice, our visualization design decisions are intended to highlight only a few key findings. First, marking the two buildings - the middle school team in light blue, and high school team in light red - highlights that most advice exchanges occur within buildings. Second, drawing the administrators in red and including arrowheads highlights that many team members go to administrators for implementation advice. Third, the arrowheads also highlight the lack of reciprocity; very few lines representing advice exchange have arrowheads at both ends. Finally, the layout choice keeps nodes spaced apart and reveals some examples of transitivity. For example, $M$ sought advice from $\mathrm{O}$, who sought advice from $\mathrm{B}$, perhaps putting $\mathrm{M}$ directly in touch with $\mathrm{B}$, who worked in a different building. Similarly, J sought advice from influential administrator $\mathrm{N}$, who sought advice from $\mathrm{D}$, perhaps putting $\mathrm{J}$ directly in touch with $\mathrm{D}$, who was otherwise relatively isolated.

\section{Conclusion}

Collecting and modeling network data require several decisions that can influence research findings, so it is important to clearly communicate these decisions in research reports. When describing network data collection, it is essential to describe how the boundary was drawn, how the relationships were defined, and how the data was collected. When describing an ERGM, it is essential to describe the model specifica- tion (i.e., which terms were included), report the estimated coefficients, and provide evidence of model fit. Clarity is also important in network visualizations, which should be included only if they clearly highlight key findings, and which should be designed with the goal of highlighting findings as clearly as possible.

Although network methods may initially appear different or complicated, they share much in common with more widely used quantitative methods. For example, network data are often collected using paper or web-based surveys, and network models are often interpreted similarly to logistic regression models. However, adopting a network approach is useful because it allows the researcher to understand participants not as 'independent observations' but as interdependent people whose relationships with one another matter. In our simple example, adopting a network approach allowed us to identify how advice-seeking relationships in the district both facilitate (e.g., by sharing advice between buildings) or hinder (e.g., by relying on just a few people for advice) the implementation of EWS. These findings could be used to further promote implementation by, for example, ensuring that EWS teams in different buildings have opportunities to interact. At the same time, network analysis does come with some unique challenges in educational settings: the data must have low levels of missingness which can be difficult particularly if buy-in from educators is low, the ERGMs must exhibit a goodness-of-fit which is not always possible from the available covariates, and the visualizations must tell a clear story which can be difficult when the networks are dense.

This brief tutorial provided a detailed example of collecting, modeling, and visualizing network data for school psychology research, but there are many options available for network research in this area. The documentation for both the statnet (Handcock et al., 2008) and igraph (Csardi et al., 2006) packages provide further details about these softwares' capabilities. Luke (2015) provides a broad overview of network analysis using R, while J. W. Neal (2020) provides a recent conceptual review of network methods throughout developmental psychology.

\section{References}

adams, j. (2020). Gathering social network data. SAGE Publications Incorporated.

Bjorklund Jr, P., \& Daly, A. J. (2021). The ties that belong: tie formation in preservice teacher identification networks. Teaching and Teacher Education, 97, 103223. https://doi.org/10.1016/j.tate.2020.103223

Borgatti, S. P., \& Cross, R. (2003). A relational view of information seeking and learning in social networks. Management science, 49(4), 432-445. https://doi.org/10.1287/mnsc.49.4.432.14428

Brown, C., \& Poortman, C. L. (2018). Networks for learning: Effective collaboration for teacher, school and system improvement. Routledge. 
Carolan, B. V. (2013). Social network analysis and education: Theory, methods $\mathcal{F}$ applications. Sage Publications.

Codding, R. S. (2021). School psychology vision for the future. School Psychology, 36, 1-5. https://doi.org/10.1037/spq0000425

Csardi, G., Nepusz, T., et al. (2006). The igraph software package for complex network research. InterJournal, complex systems, 1695(5), 1-9.

Faria, A.-M., Sorensen, N., Heppen, J., Bowdon, J., Taylor, S., Eisner, R., \& Foster, S. (2017). Getting students on track for graduation: Impacts of the early warning intervention and monitoring system after one year. rel 2017-272. Regional Educational Laboratory Midwest.

Handcock, M. S., Hunter, D. R., Butts, C. T., Goodreau, S. M., \& Morris, M. (2008). statnet: Software tools for the representation, visualization, analysis and simulation of network data. Journal of statistical software, 24(1), 1548. https://doi.org/10.18637/jss.v024.i01

Harrigan, N. M., Labianca, G. J., \& Agneessens, F. (2020). Negative ties and signed graphs research: Stimulating research on dissociative forces in social networks. Soc. Networks, 60, 1-10. https://doi.org/10.1016/j.socnet.2019.09.004

Holland, P. W., \& Leinhardt, S. (1973). The structural implications of measurement error in sociometry. Journal of Mathematical Sociology, 3(1), 85-111. https://doi.org/10.1080/0022250X.1973.9989825

Huisman, M. (2009). Imputation of missing network data: Some simple procedures. Journal of Social Structure, 10(1), 1-29.

Kitts, J. A., \& Leal, D. F. (2021). What is (n't) a friend? dimensions of the friendship concept among adolescents. Social Networks, 66, 161-170. https://doi.org/10.1016/j.socnet.2021.01.004

Krause, R. (2019). Multiple imputation for missing network data.

Laumann, E. O., Marsden, P. V., \& Prensky, D. (1989). The boundary specification problem in network analysis. Research methods in social network analysis, 61, 87.

Luke, D. A. (2015). A user's guide to network analysis in $r$. Springer.

McPherson, M., Smith-Lovin, L., \& Cook, J. M. (2001). Birds of a feather: Homophily in social networks. Annual review of sociology, 27(1), 415-444. https://doi.org/10.1146/annurev.soc.27.1.415

Neal, J. W. (2008). "kracking" the missing data problem: applying krackhardt's cognitive social structures to school-based social networks. Sociology of Education, 81(2), 140-162. https://doi.org/10.1177/003804070808100202

Neal, J. W. (2020). A systematic review of social network methods in high impact developmental psychology journals. Social Development, 29(4), 923-944. https://doi.org/10.1111/sode.12442

Neal, J. W., Neal, Z. P., Barrett, C. A., \& Brutzman, B. (2020). Are principals' social networks associated with interventions' social validity? School Mental Health, 12(4), 812825. https://doi.org/10.1007/s12310-020-09388-5

Neal, J. W., \& Veenstra, R. (2021). Network selection and influence effects on children's and adolescents' internalizing behaviors and peer victimization: a systematic review. Developmental Review, 59. https://doi.org/10.1016/j.dr.2020.100944
Neal, Z., Neal, J. W., \& Piteo, A. (2020). Call me maybe: using incentives and follow-ups to increase principals' survey response rates. Journal of Research on Educational Effectiveness, 13(4), 784-793. https://doi.org/10.1080/19345747.2020.1772423

Neal, Z. P., \& Neal, J. W. (2022). That'll move the chains: Collecting network chain data. Social Networks. https://doi.org/10.1016/j.socnet.2019.10.007

O’Brennan, L., Pas, E., \& Bradshaw, C. (2017). Multilevel examination of burnout among high school staff: Importance of staff and school factors. School Psychology Review, 46(2), 165-176. https://doi.org/10.17105/SPR-2015-0019.V46-2

R Core Team. (2020). R: A language and environment for statistical computing [Computer software manual]. Vienna, Austria. Retrieved from https://www.R-project.org/

Robins, G., Pattison, P., Kalish, Y., \& Lusher, D. (2007). An introduction to exponential random graph ( $\left.\mathrm{p}^{*}\right)$ models for social networks. Social networks, 29(2), 173-191. https://doi.org/10.1016/j.socnet.2006.08.002

Sijtsema, J. J., \& Lindenberg, S. M. (2018). Peer influence in the development of adolescent antisocial behavior: Advances from dynamic social network studies. Developmental Review, 50, 140-154. https://doi.org/10.1016/j.dr.2018.08.002

Snijders, T. A. (2017). Stochastic actor-oriented models for network dynamics. Annual Review of Statistics and Its Application, 4, 343-363. https://doi.org/10.1146/annurev-statistics060116-054035

Therriault, S. B., O'Cummings, M., Heppen, J., Yerhot, L., \& Scala, J. (2017). Early warning intervention and monitoring system implementation guide. Michigan Department of Education.

U.S. Department of Education. (2016). Issue Brief: Early warning systems. 
Appendix A. Replication code and output

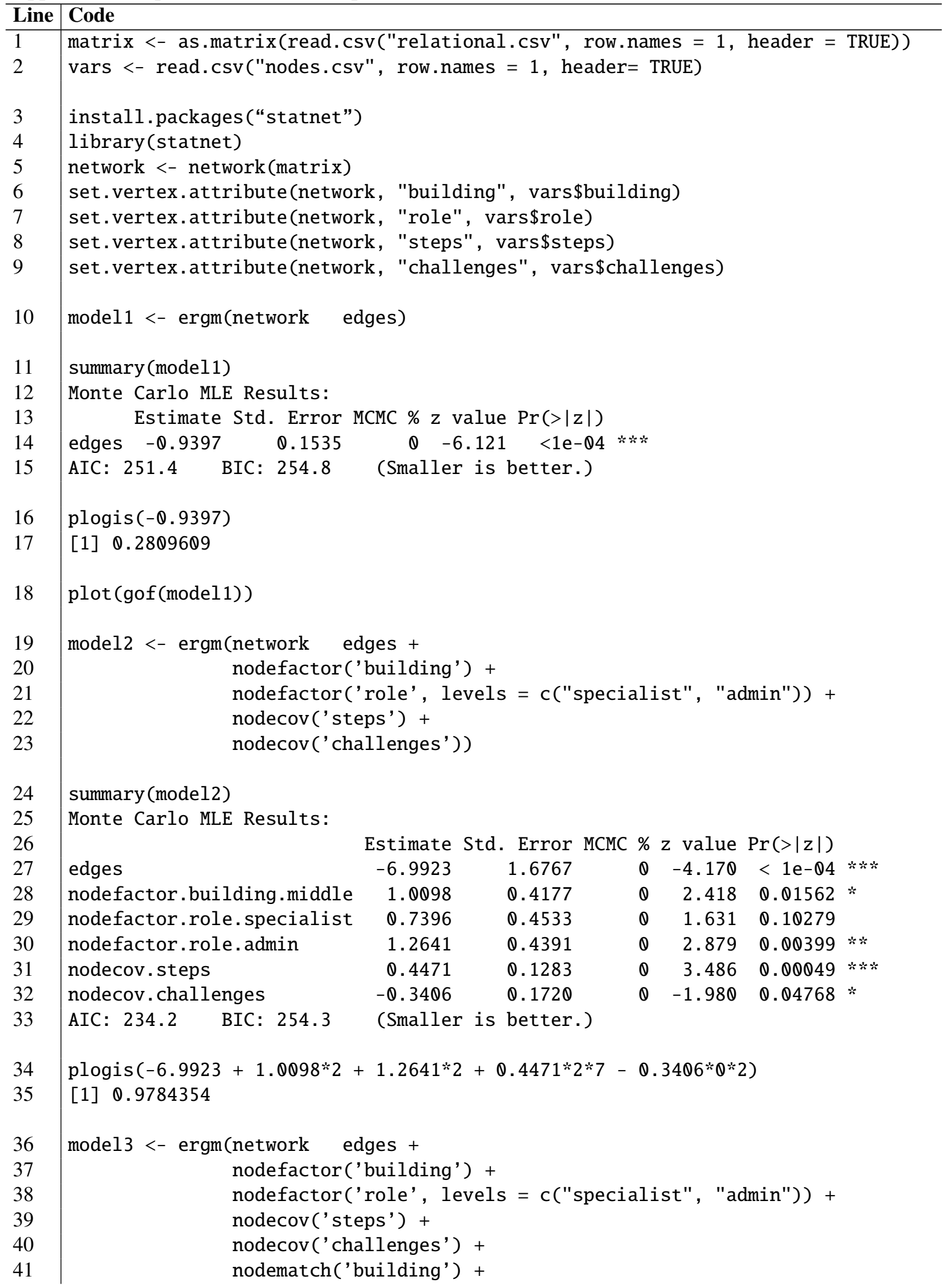


Appendix A. Replication code and output (continued)

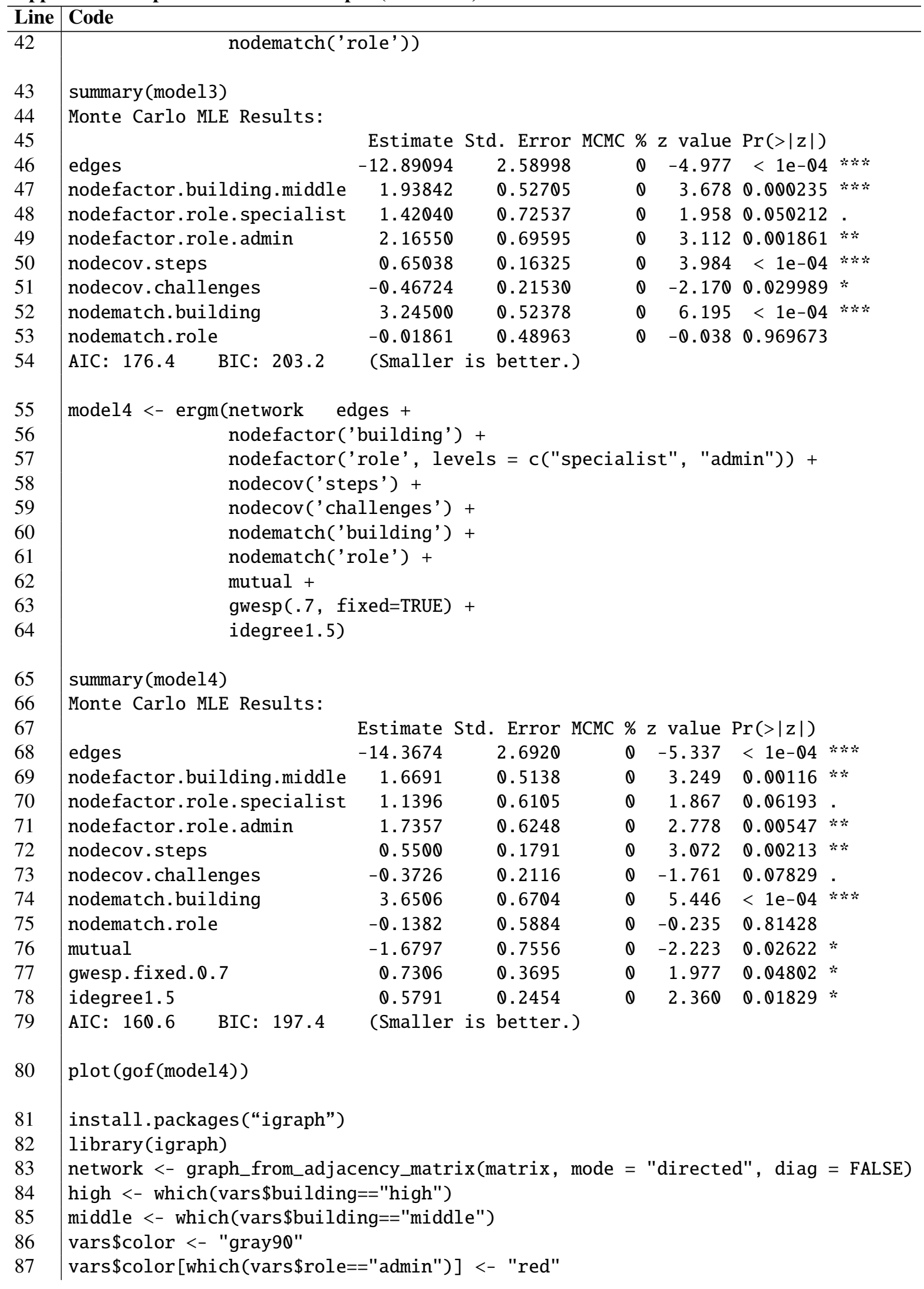


Appendix A. Replication code and output (continued)

\begin{tabular}{|c|c|}
\hline Line & Code \\
\hline 88 & plot (network, \\
\hline 89 & mark groups $=$ list $($ high, middle $)$, \\
\hline 90 & edge. $\operatorname{col}$ or $=\operatorname{rgb}(\theta, 0,0, .33)$ \\
\hline 91 & edge. arrow. size $=.5$ \\
\hline 92 & vertex. color $=$ vars $\$$ color \\
\hline 93 & vertex.size $=$ degree $($ network $)+5$, \\
\hline 94 & layout = layout_with_kk, \\
\hline 95 & mark . border $=\mathrm{NA}$ \\
\hline 96 & vertex frame. color $=\mathrm{NA}$ ) \\
\hline
\end{tabular}

